\title{
Modeling and simulation of flexible axially moving arm
}

\author{
Ruixia Liu \\ Institute of Mechanics, Chinese Academy of Sciences \\ School of Engineering Science, University of Chinese Academy \\ of Sciences \\ Beijing, China \\ liuruixia@imech.ac.cn
}

\author{
Jie Zhang* \\ School of Mechanical and Materials Engineering, North China \\ University of Technology \\ Beijing, China \\ zhangjie@ncut.edu.cn (Corresponding author)
}

\begin{abstract}
A finite element method is developed for modeling and simulation of flexible axially moving arm, considering the effects of Coulomb friction, the elasticity of the entire arm and the clearances between the arm and joint. Mechanical interactions between the arm and joint are simulated by a model which is formulated based on the penalty method. The the contact forces are calculated based on the contact model, trialand-error algorithms. Based on the obtained contact forces, the system dynamic equations are numerically solved by Newmark method. A numerical example is simulated to show the capability of the new method. The obtained results also reveal that it's inappropriate to ignore the influences of friction force and the small deformation of the arm part inside the joint. The method should prove useful in designing and controlling mechanical systems with axially moving arm.
\end{abstract}

Keywords-axially moving arm, coulomb friction, penalty method

\section{INTRODUCTION}

Axially moving arm is used widely in many engineering fields, such as hoisting equipment, loading vehicle and industrial robot [1]. In recent years, researchers began to pay attention to the application of axially moving arm in space robotics, for instance, the Next Generation Canadarm program [2].

Many researches have been conducted about the arm axially translating through a stationary prismatic joint [3]. The problem becomes more complex as the arm sliding through a rotating-prismatic joint. Al-Bedoor and Khulief [4] studied the dynamic response of a flexible arm translating inside a rotatingprismatic joint based on FEM. Korayem et al. [5] formulated a systematical method to derive the dynamic equations of the motion of $\mathrm{N}$-flexible link manipulators with rotating-prismatic joints. Kalyoncu [6] analyzed the modeling and simulation of a flexible robot manipulator with rotating-prismatic joint.

However, in most studies related to axially moving arm, the sliding motion of the arm in prismatic joint is assumed frictionless (perfect slip condition) and the arm part which is embedded in the joint is assumed to be rigid. Actually, the interaction between the prismatic joint and the sliding arm is probably to be more complicated, the influence of friction, clearance and small deformations of the arm part inside the joint is significant which can cause non-negligible effects on the system dynamics.
This paper proposed a FEM to analyze the dynamic response of the sliding arm translating in a rotating-prismatic joint with Coulomb friction and the clearance between the joint and arm, the entire arm is assumed flexible, including the arm part inside the joint. In terms of the penalty method, kinetoelasto-dynamic (KED) method and trial-and-error algorithms, a FE method for calculating contact forces is put forward which can be used for both the stick and slip states. Then the contact forces are applied on the arm as external forces, effects of inertia forces and axial shortening of arm are taken into consideration in the model, the FEM dynamic Equations are solved in terms of Newmark method. The simulation results of the proposed method reveals the capability of this method and it will be beneficial for design and control of the mechanical systems with axially moving arm.

\section{Model AND Simulation Method}

Fig. 1 shows an axially sliding arm moves inside a rotatingprismatic joint, it can be treated as flexible system with multiunilateral constraints. The interactions between the arm and the joint are complicated due to the clearance and Coulomb friction forces between joint guides and the arm part embedded in the joint. A contact model is developed to describe the interactions between the arm and the joint. As indicated in Fig. 1, the upper and lower surface of the arm are defined as surface $(+)$ and surface $(-)$ respectively, the symbol " $(+)$ " and " $(-)$ " denote the physical quantities of the nodes on the surface $(+)$ and $(-)$. The normal and tangential directions are defined along $x^{\prime}$-axis and $\mathrm{y}^{\prime}$-axis respectively, the symbol "N" and "T" denote them respectively.

Coulomb friction is considered in this research, which is often utilized to describe dry friction. With consideration of Coulomb friction, multiple nodal friction force conditions at the nodes on the contact surfaces of the arm have to be determined.

In penalty method, the mechanical interactions are modeled by introducing fictitious linear springs which are appended on the nodes of contact surfaces, the contact forces are proportional to the quantity of penetration by introducing a penalty factor, which equals the stiffness of the springs. The FE implementation of the penalty method is discussed in detail in textbook. 


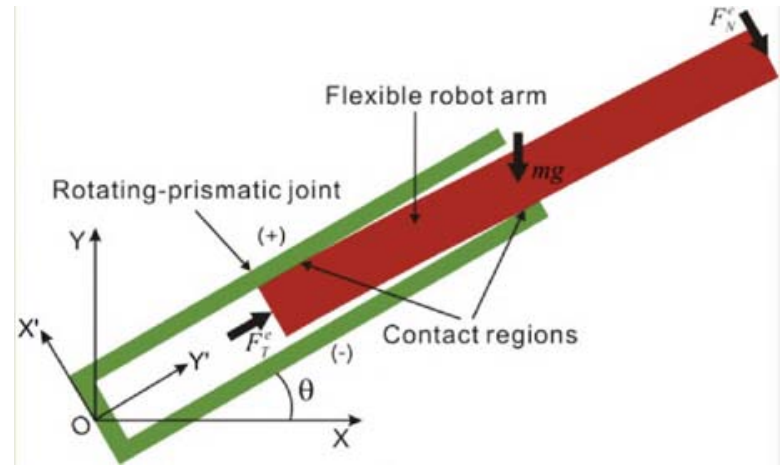

Fig.1 $\Lambda$ xially moving arm with rotating-prismatic joint.

Fictitious springs have been used for numerical simulation in several contact problems. Based on the penalty method, guide surfaces of rotating-prismatic joint are modeled by applying a number of fictitious linear springs in normal and tangential directions, similar to the Winkler surface model of elastic foundation theory with normal fictitious springs appended on the surface. It is worth noting that a great number of microscopic linear springs can simulate the macroscopic nonlinear phenomenon, such as the nonlinear relationship between the contact force and elastic displacement.

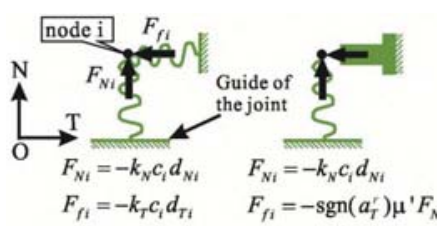

(a)

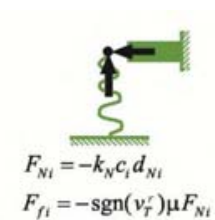

(c)

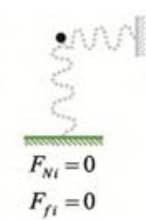

(d)
Fig. 2 Contact models for modeling nodal contact states.

In terms of the penalty method, four nodal contact models are developed for modeling nodal contact states of frictional contact problem. The models are used to describe the nodal contact forces during the motion of the sliding arm, as illustrated in Fig. 2.

These springs are linear and fictitious, the green rectangles manifest the tangential nodal friction forces $F_{f i}$ is proportional to the normal nodal contact forces $F_{N i} c_{i}$ is contact coefficient of node $i$, which is determined by trial-and-error methods.

For the normal nodal contact forces, the contact model is described as a linear function between the normal nodal contact force $F_{N i}$ and normal nodal displacement $d_{N i}$, as described in Fig. 2. Where $i$ is the number of the contacted node, $K_{N}$ is stiffness coefficient of normal springs.

For the tangential nodal friction forces, contact model A indicates the tangential nodal friction force $F_{f i}$ is set as a linear function of the tangential nodal displacement $\boldsymbol{d}_{T i}$ when the nodal friction force is smaller than the maximum static friction force of node $i$ during sticking, as illustrated in Fig. 2(a). Where $K_{T}$ is stiffness coefficient of tangential springs

Contact model $\mathbf{B}$ manifests the nodal friction force reaches its maximum during sticking, the maximum static friction force of the node is expressed as a linear function of the normal nodal contact force, as illustrated in Fig. 2(b).
In contact model $\mathrm{C}$, nodal friction force is linear to normal nodal contact force at contact nodes during slipping, as described in Fig. 2(c).

As shown in Fig. 2(d), contact model D indicates the node is out of contact with the guide surfaces, the contact coefficient equals zero and the springs are drawn as gray dashed line

The accuracy of penalty method relies on the appropriate choice of the stiffness of fictitious springs, which defined as penalty factor and covered a large range of values [28]. A too small penalty factor will cause large penetrations and errors into the solution, but a large value may introduce numerical difficulties of the convergence of the iteration, especially in contact problems using FEM [27]. In this paper, varying penalty factor is adopted to make sure the convergence of iteration and numerical accuracy $[27,29]$.

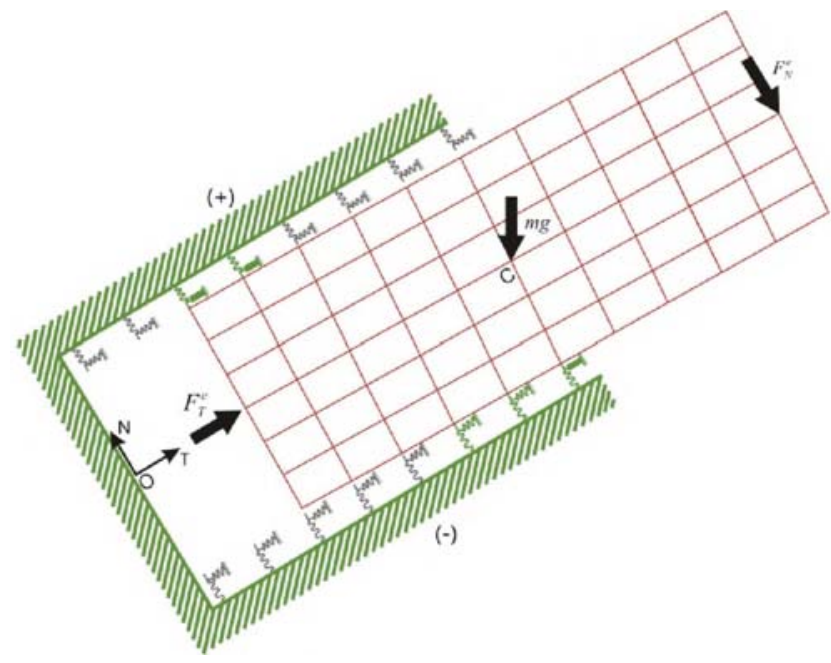

Fig. 3 Diagram of contact model for sticking.

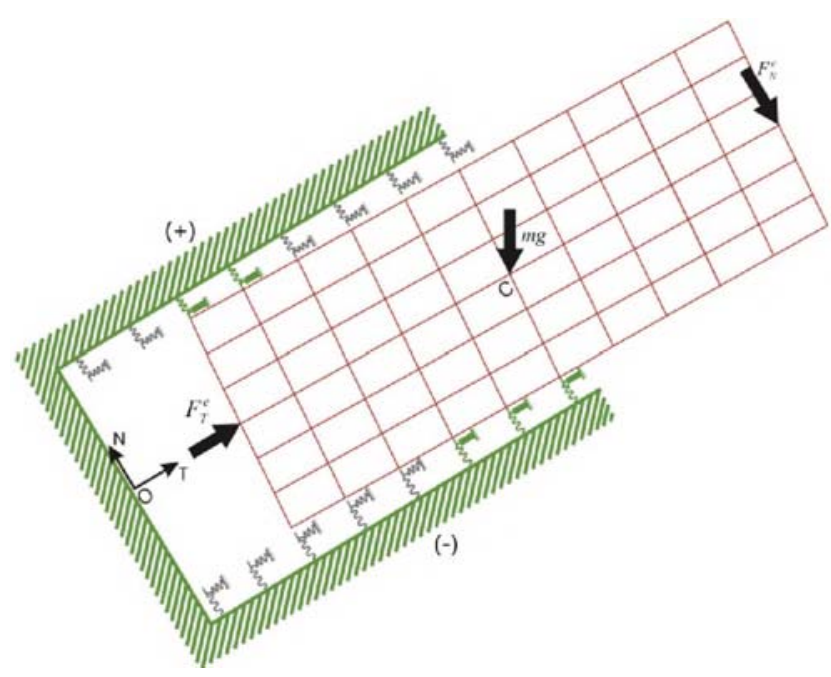

Fig.4 Diagram of contact model for slipping.

According to the nodal contact force models and FEM, the models of axially moving arm are developed as described in Figs. 3 and 4. Springs are applied between contact nodes of the arm and guide surfaces of the joint to simulate the interaction between the arm and the joint. These springs are independent to 
each other, they are fictitious and just for numerical simulation. The stiffness of these fictitious springs depends on geometric properties, material properties and requirement of convergence, and can be determined by the theory of penalty method [30].

When the arm moves inside the rotating-prismatic joint, contact model $\mathrm{A}, \mathrm{B}$ and $\mathrm{D}$ will occur at the nodes on the surfaces simultaneously during sticking, as described in Fig. 3; during slipping, contact model $\mathrm{C}$ and $\mathrm{D}$ will occur simultaneously as illustrated in Fig. 4. Trial-and-error algorithms are developed to determine the contact force models for each node at each time step, which are illustrated.

Based on FEM theory, contact force vector and deformation displacement vector can be obtained, according to the contact force model of the arm, the relationship between contact force vector $F_{c}$ and deformation displacement vector $d$ can be illustrated as $F_{c}=K_{c} d . K_{c}$ is the contact stiffness matrix of axially moving arm with time-varying components, due to the change of nodal contact states during the motion of arm. Trialand-error methods are proposed for determining nodal contact states of the arm.

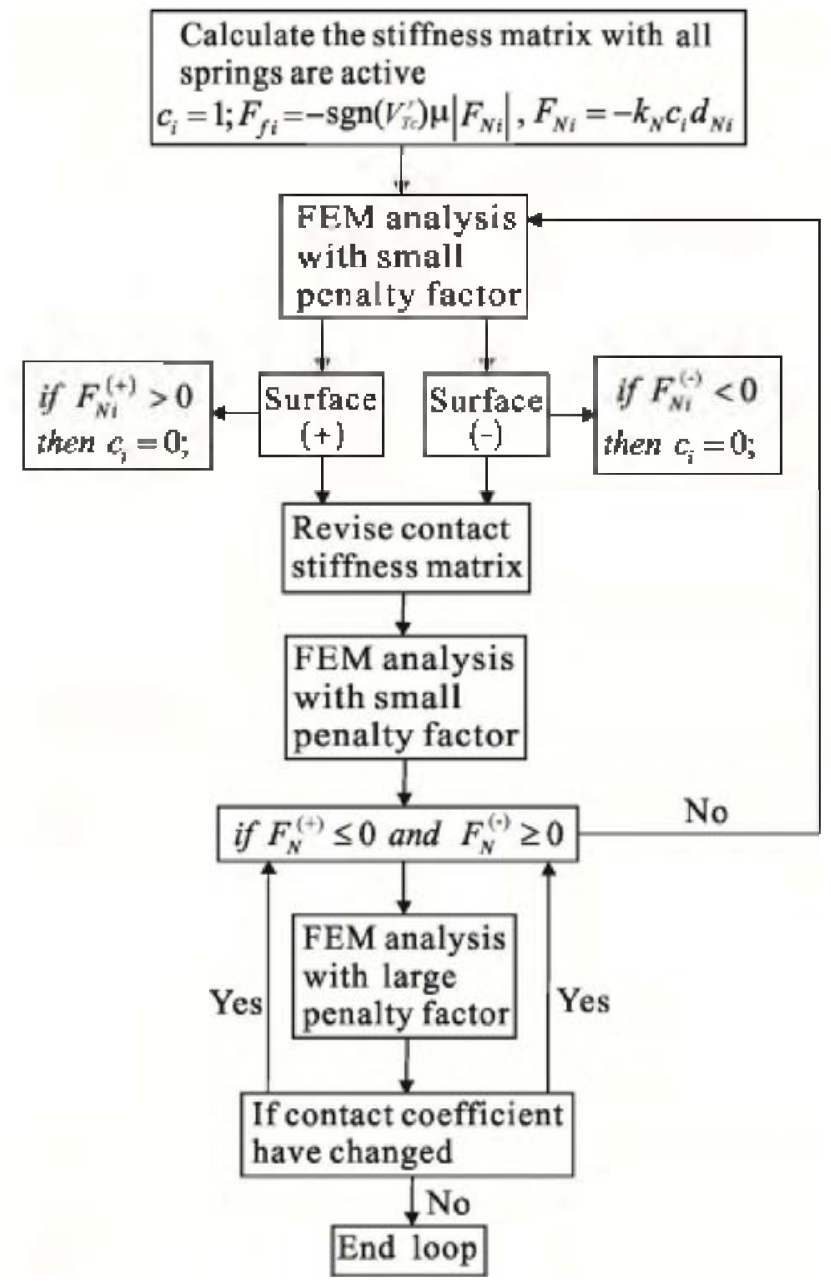

Fig.5 Block diagram of the trial-and-error algorithm.
Based on the contact model, the trial-and-error method is proposed to determine nodal contact states, as illustrated in Fig. 5.

The coordinate systems of axially moving arm are shown in Fig. 1, due to the joint's rotation, any node of the arm described in the relative coordinate system Ox'y' will move to a new position in the absolute reference coordinate system Oxy. When the arm moves inside the rotating-prismatic joint, it will be influenced by gravity, Coriolis force, convected inertial force, external forces and contact forces between the arm and joint. According to the contact force model illustrated above, the dynamic equations of the axially moving arm can be obtained.

In the numerical solution methods for FEM dynamic analysis, such as Newmark method, an essential problem is the oscillations arisen in accelerations. In normal studies, the solution procedure is relatively easy to converge to a stable solution. However, as to the mechanical system with multiunilateral constraints and Coulomb friction, the nonlinearities introduced by time-varying contacted regions and contact forces will bring great difficulties in convergence.

KED method provides a convenient vehicle for avoiding the oscillations in the acceleration [31, 32]. In terms of the KED method, trial-and-error method and Newmark method, a numerical algorithm for frictional axially moving arm with is described which can converge to a stable solution [27]. At First, according to KED method, the static FEM analysis is conducted by applying inertial forces as external forces

The static FEM analysis and trial-and-error methods are implemented to solve dynamic equation and calculated the contact forces $\mathbf{F}^{c}$, then a new relative acceleration and inertial force can be approximated based on $\mathbf{F}^{c}$, after that the static FEM analysis is re-conducted based on the new relative acceleration and inertial force. Repeat this cycle continuously until the desired accuracy of acceleration is reached. At last, the Newmark method is implemented to solve the forced vibration system equations by utilizing the final nodal contact forces as external forces.

When the arm is in the state of stick, there is no relative motion and no tendency to relative motion, relative velocity and acceleration are both zero, only exist micro-slip caused by deformations, the static FEM analysis equations is described as a linear algebraic system, which can be easily solved without iteration.

\section{NUMERICAL RESULATS AND DISCUSSION}

A numerical example is solved for examining the effectiveness of the method developed for the frictional flexible axially moving arm system with rotating-prismatic joint. As illustrated in Fig. 1, in the example, the guides of the rotatingprismatic joint are rigid and the entire arm is flexible. The material damping is ignored and the mass center of each body is assumed to be located at the geometric center of the body. In order to verify the effectiveness of the method and demonstrate the influences of the friction and rotation on the system responses, different angular velocities of the rotating-prismatic joint are analyzed 
The sum of friction forces acted on the arm is shown in Fig. 6. As illustrated in Fig. 6 at first the arm is in the state of stick and friction forces grow with the external tangential forces, then the external tangential forces exceeds the sum of nodal maximum static friction forces, stick-slip transition occurs and results in the mutation of friction forces. It can be observed that the larger the angular velocity is, the earlier the arm will start to move outwards, due to the convected inertial force.

When considering the small deformation of the arm, the contact forces between the arm and the joint are distributed over a contacted region, and the moment arms of external forces and normal nodal contact forces about moment center of the arm are influenced by the motion of the arm. Based on the moment equilibrium principle and the linear relationship between tangential and normal nodal contact forces, the absolute value of normal and tangential contact forces will vary due to the deformation of the arm. Based on the analysis, we can draw the conclusion that the values of the nodal contact forces will be varied as the motion of the arm, if the external moment keeps constant, the larger the extended lengths of arm is, the larger the friction force will be.

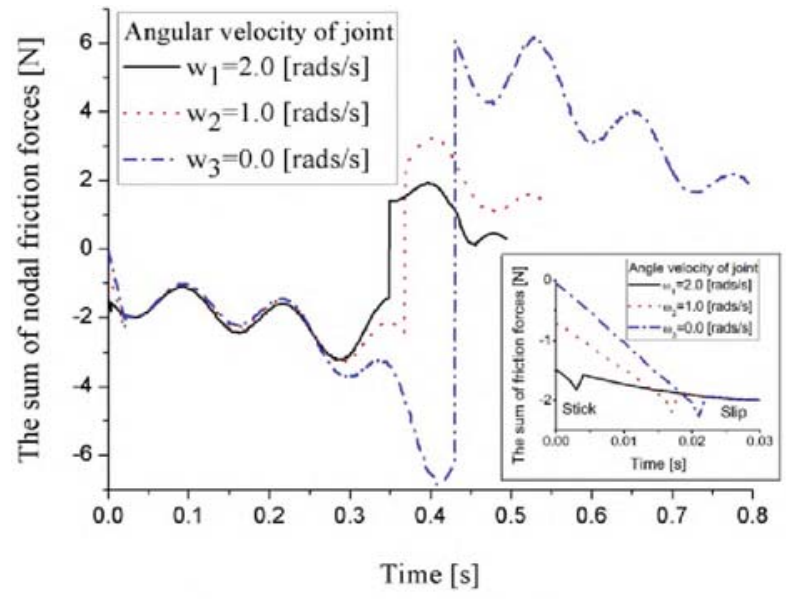

Fig.6 The sum of nodal friction forces.

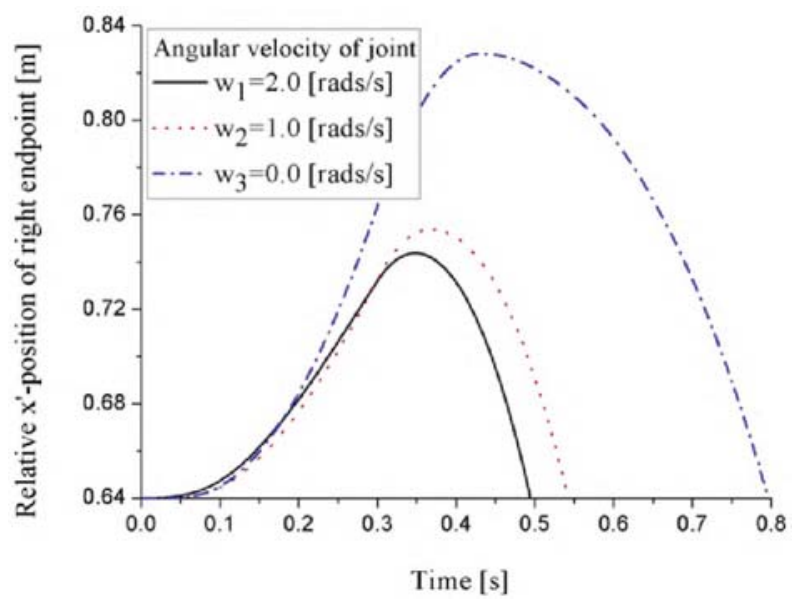

Fig. 7 Absolute x-position of right endpoint of axially moving arm.
The absolute value of friction forces vibrates with the sum of normal external forces with the same frequency, and its total variation tendency is to rise as the arm moving outwards and is to decline as the arm moving inwards. Due to the components of gravity in $x^{\prime}$ and $y^{\prime}$ directions vary as the increase of the rotation angle, it shows significantly that the joint angular velocity influences the absolute value of friction forces obviously.

The absolute x-position of the right endpoint of the arm are plotted in Fig. 7. It can be seen that the results obtained based on different values of angular velocity of rotating-prismatic joint have different trajectories with significant distinction.

\section{CONCLUSIONS}

A finite element method is developed for the analysis of flexible axially moving arm with frictional rotating-prismatic joint, considering friction, flexibility of the arm part inside the joint, clearance and multi-unilateral constraints. The numerical simulations have demonstrated the capability of the method. The method will be beneficial for design and control of the mechanical systems with axially moving arm.

\section{ACKNOWLEDGEMENTS}

This research was supported by the National Natural Science Foundation of China [grant number 11702294] and the Scientific Research Foundation of North China University of Technology.

\section{REFERENCES}

[1] A. Flores-Abad, O. Ma, K. Pham, et al. "A review of space robotics technologies for on-orbit servicing", Progress in aerospace sciences, vol.68 2014, pp. 1-26. doi: 10.1016/j.paerosei.2014.03.002.

[2] S.K. Dwivedy, R. Mukherji, C. Lyn, et al. "On the Application of Robotics to On-Orbit Spacecraft Servicing-The Next Generation Canadarm Project", Proceedings of 11 th Intl. Symposium on Artificial Intelligence, Robotics and Automation in Space, 2012, pp. 3-7.

[3] S.K. Dwivedy, P. Eberhard, "Dynamic analysis of flexible manipulators, a literature review", Mechanism and Machine Theory, vol. 41(7), 2006, pp. 749-777. doi: 10.1016/j.mechmachtheory.2006. 01.014

[4] B.O. Al-Bedoor, Y.A. Khulief, "Finite element dynamic modeling of a translating and rotating flexible link", Computer Methods in Applied Mechanics and Engineering, vol, 131, 1996, pp. 173-189. doi: 10.1016/0045-7825(95)00968-X.

[5] M.H. Korayem, A.M. Shafei, S.F. Dehkordi, "Systematic modeling of a chain of N-flexible link manipulators connected by revolute-prismatic joints using recursive Gibbs-Appell formulation", Archive of applied mechanics, Vol, 84(2), 2014, pp. 187-206. doi: 10.1007/ s00419-0130793-y

[6] M. Kalyoncu, "Mathematical modelling and dynamic response of a multi-straight-line path tracing flexible robot manipulator with rotatingprismatic joint", Appllied Mathematical Modelling, vol. 32(6), 2008, pp. 1087- 1098. doi: 10.1016/j.apm.2007.02.032 